\title{
STOCHASTIC DOMINANCE THEORY FOR LOCATION-SCALE FAMILY
}

\author{
WING-KEUNG WONG
}

Received 17 January 2006; Revised 1 August 2006; Accepted 2 August 2006

Meyer (1987) extended the theory of mean-variance criterion to include the comparison among distributions that differ only by location and scale parameters and to include general utility functions with only convexity or concavity restrictions. In this paper, we make some comments on Meyer's paper and extend the results from Tobin (1958) that the indifference curve is convex upwards for risk averters, concave downwards for risk lovers, and horizontal for risk neutral investors to include the general conditions stated by Meyer (1987). We also provide an alternative proof for the theorem. Levy (1989) extended Meyer's results by introducing some inequality relationships between the stochasticdominance and the mean-variance efficient sets. In this paper, we comment on Levy's findings and show that these relationships do not hold in certain situations. We further develop some properties among the first- and second-degree stochastic dominance efficient sets and the mean-variance efficient set.

Copyright (c) 2006 Wing-Keung Wong. This is an open access article distributed under the Creative Commons Attribution License, which permits unrestricted use, distribution, and reproduction in any medium, provided the original work is properly cited.

\section{Introduction}

Mean-variance (MV) efficient sets have been widely used in both economics and finance to analyze how people make their choices among risky assets. Markowitz [21] demonstrated that if the ordering of alternatives is to satisfy the von Neumann-Morgenstern [39] (NM) axioms of rational behavior, only a quadratic (NM) utility function is consistent with an ordinal expected utility function that depends solely on the mean and variance of the return. Thereafter, Feldstein [7], Hanoch and Levy [12], Rothschild and Stiglitz [31,32], and others commented that the MV criterion is applicable only when the decision maker's utility function is quadratic and the probability distribution of return is normal. Moreover, Baron [2] pointed out that even if the return for each alternative has a normal distribution, the MV framework cannot be used to rank alternatives consistently 
with the NM axioms unless a quadratic NM utility function is specified. Meyer [25] extended the MV theory to include general utility functions and comparison between distributions that differ only by location and scale parameters.

Meyer's extensions are important as it is well known that the distribution of investment returns is usually nonnormal and the restriction of the utility function to the quadratic form is too limited in scope. These restrictions were popular in the literature not only before Meyer's findings but remained common after Meyer's findings. For example, Zhao and Ziemba [45] restricted the use of mean-variance criterion to normal or log-normal distributions and the quadratic utility function. Chow [4] pointed out that the meanvariance portfolio theory assumes that investor utility functions are quadratic and/or the return distributions of assets are multivariate normal. In this paper, we make some comments on Meyer's paper and extend the results from Tobin [37], who postulated that the indifference curve is convex upwards for risk averters and is concave downwards for risk lovers, to include a wide family of distributions for the returns as well as to include general utility functions as stated in Meyer [25]. We also provide an alternative proof for the theorem.

Levy [16] extended Meyer's results to prove that the first- (FSD) and second-degree (SSD) stochastic dominance efficient sets are equal to the mean-variance (MV) efficient set under certain conditions and established some inequality relationships between the variables in the same location-scale family. In this paper, we comment on Levy's findings and show that the inequality relationships developed by Levy do not hold in certain situations. We further explore the relationships among the FSD, SSD, and MV efficient sets, which culminate in three important findings: (1) the SSD efficient set is a proper subset of the FSD efficient set, (2) the SSD efficient set is a proper subset of the MV efficient set, and (3) the FSD efficient set is not equal to the MV efficient set in a way that neither is a proper subset of each other.

Being of both theoretical and practical interests, the main challenge of the MV and SD analyses is to identify the assets that constitute attainable efficient portfolios. Unfortunately, the relationships between the MV efficient sets and the SD efficient sets have not been well established. With this in mind, we seek to develop the relationships between the MV and SD efficient sets to capture the essence of portfolio selection here. In addition, we explore the shapes of indifference curves for risk averters, risk lovers, and risk-neutral investors. Our findings could be useful in facilitating the MV and SD procedures and enabling investors to make wiser decisions in their investments.

We begin by introducing a brief literature in this section. In Section 2, we first review, discuss, and give comments on some properties stated in Meyer [25], Levy [16], and Sinn [34]. We then proceed to develop some properties on the expected utility maximization and the stochastic dominance theory for the location-scale family. The concluding remarks are in Section 3.

\section{Theory}

In this section, we first review and discuss some properties stated in Meyer [25], Levy [16], and Sinn [34], and further extend their work by developing some additional properties. 
In order to avoid confusion, we use "proposition" to state our results and "property" to state the results produced by Meyer [25] and Levy [16].

Let the return $X$ be the random variable with zero mean and variance one, with the location-scale family $\mathscr{D}$ generated by $X$ such that

$$
\mathscr{D}=\{Y \mid Y=\mu+\sigma X,-\infty<\mu<\infty, \sigma>0\}
$$

The expected utility $V(\sigma, \mu)$, see Meyer [25], for the utility $U$ on the random variable $Y$ can then be expressed as

$$
V(\sigma, \mu)=E[U(Y)]=\int_{a}^{b} u(\mu+\sigma x) d F(x)
$$

where $[a, b]$ is the support of $X, F$ is the distribution function of $X$, and the mean and variance of $Y$ are $\mu$ and $\sigma^{2}$, respectively. We note that the requirement of the zero mean and unit variance for $X$ is not necessary. However, without loss of generality, we can make these assumptions as we will always be able to find such a seed random variable in the location-scale family.

For any constant $\alpha$, the indifference curve drawn on the $(\sigma, \mu)$ plane such that $V(\sigma, \mu)$ is a constant can be expressed as

$$
C_{\alpha}=\{(\sigma, \mu) \mid V(\sigma, \mu) \equiv \alpha\}
$$

In the indifference curve, we follow Meyer [25] to have

$$
V_{\mu}(\sigma, \mu) d \mu+V_{\sigma}(\sigma, \mu) d \sigma=0
$$

or

$$
V_{\mu}(\sigma, \mu) \frac{d \mu}{d \sigma}+V_{\sigma}(\sigma, \mu)=0
$$

where

$$
\begin{gathered}
V_{\mu}(\sigma, \mu)=\frac{\partial V(\sigma, \mu)}{\partial \mu}=\int_{a}^{b} u^{\prime}(\mu+\sigma x) d F(x), \\
V_{\sigma}(\sigma, \mu)=\frac{\partial V(\sigma, \mu)}{\partial \sigma}=\int_{a}^{b} u^{\prime}(\mu+\sigma x) x d F(x) .
\end{gathered}
$$

The following proposition is then obtained by applying Meyer [25, Properties 1 and 2] and the implicit function theorem.

Proposition 2.1. If the distribution function of the return with mean $\mu$ and variance $\sigma^{2}$ belongs to a location-scale family and for any utility function $u$, if $u^{\prime}>0$, then the indifference curve $C_{\alpha}$ can be parameterized as $\mu=\mu(\sigma)$ with slope

$$
S(\sigma, \mu)=-\frac{V_{\sigma}(\sigma, \mu)}{V_{\mu}(\sigma, \mu)} .
$$


4 Stochastic dominance theory for location-scale family

In addition,

(1) if $u^{\prime \prime} \leq 0$, then the indifference curve $\mu=\mu(\sigma)$ is an increasing function of $\sigma$; and

(2) if $u^{\prime \prime} \geq 0$, then the indifference curve $\mu=\mu(\sigma)$ is a decreasing function of $\sigma$.

Proof. From (2.6), we have

$$
S(\sigma, \mu)=-\frac{\int_{a}^{b} u^{\prime}(\mu+\sigma x) x d F(x)}{\int_{a}^{b} u^{\prime}(\mu+\sigma x) d F(x)}
$$

in which $\int_{a}^{b} u^{\prime}(\mu+\sigma x) d F(x)>0$ because $u^{\prime}>0$. For the numerator, as $E(X)=0$, we have $\int_{a}^{0} x d F(x)=-\int_{0}^{b} x d F(x)$. If $u^{\prime \prime}<0$, we have

$$
\begin{aligned}
\int_{0}^{b} u^{\prime}(\mu+\sigma x) x d F(x) & <\int_{0}^{b} u^{\prime}(\mu) x d F(x)=-\int_{a}^{0} u^{\prime}(\mu) x d F(x) \\
& <-\int_{a}^{0} u^{\prime}(\mu+\sigma x) x d F(x)
\end{aligned}
$$

Hence, $S(\sigma, \mu)>0$. Similarly, if $u^{\prime \prime}>0$, we have $S(\sigma, \mu)<0$.

Meyer [25] continued to investigate the properties of $\partial S(\sigma, \mu) / \partial \mu$ without the restriction of $V(\sigma, \mu) \equiv \alpha$ and obtained the following property (we refer to Property 5 in Meyer's paper).

Property 2.2. $\partial S(\sigma, \mu) / \partial \mu \leq(=\geq) 0$ for all $\mu$ and for all $\sigma \geq 0$ if and only if $u(\mu+\sigma x)$ displays decreasing (constant, increasing) absolute risk aversion.

We note that Sinn [34] obtained similar results as the above property in Meyer's paper. But similar to Meyer's approach, the proof of the results in Sinn [34] was also done without the restriction of $V(\sigma, \mu) \equiv \alpha$. It should be equally important to study the convexity of the indifference curve $C_{\alpha}$ with the restriction of $V(\sigma, \mu) \equiv \alpha$. Under the constraint of $(\sigma, \mu) \in C_{\alpha}$, we have the following proposition for $\partial S(\sigma, \mu) / \partial \sigma$ as a complement of Meyer's Property 5 and Sinn's work.

Proposition 2.3. The distribution function of the return with mean $\mu$ and variance $\sigma^{2}$ belongs to a location-scale family. For any utility function $u$ with $u^{\prime}>0$,

(1) if $u^{\prime \prime} \leq 0$, then $\mu=\mu(\sigma)$ is a convex function of $\sigma$, and

(2) if $u^{\prime \prime} \geq 0$, then $\mu=\mu(\sigma)$ is a concave function of $\sigma$.

Proof. As

$$
\frac{d \mu}{d \sigma}=-\frac{\int_{a}^{b} u^{\prime}(\mu+\sigma x) x d F(x)}{\int_{a}^{b} u^{\prime}(\mu+\sigma x) d F(x)}=-\frac{I_{1}}{I_{2}},
$$


we have

$$
\begin{aligned}
\frac{d^{2} \mu}{d \sigma^{2}} & =\frac{1}{I_{2}^{2}}\left(I_{1} \frac{\partial I_{2}}{\partial \sigma}-I_{2} \frac{\partial I_{1}}{\partial \sigma}\right) \\
& =\frac{I_{1}}{I_{2}^{2}} \int_{a}^{b} u^{\prime \prime}(\mu+\sigma x)\left(\frac{d \mu}{d \sigma}+x\right) d F-\frac{1}{I_{2}} \int_{a}^{b} u^{\prime \prime}(\mu+\sigma x)\left(\frac{d \mu}{d \sigma}+x\right) x d F \\
& =-\frac{1}{I_{2}} \frac{d \mu}{d \sigma} \int_{a}^{b} u^{\prime \prime}(\mu+\sigma x)\left(\frac{d \mu}{d \sigma}+x\right) d F-\frac{1}{I_{2}} \int_{a}^{b} u^{\prime \prime}(\mu+\sigma x)\left(\frac{d \mu}{d \sigma}+x\right) x d F \\
& =-\frac{1}{I_{2}} \int_{a}^{b} u^{\prime \prime}(\mu+\sigma x)\left(\frac{d \mu}{d \sigma}+x\right)^{2} d F \\
& =-\frac{\int_{a}^{b} u^{\prime \prime}(\mu+\sigma x)(d \mu / d \sigma+x)^{2} d F}{\quad \int_{a}^{b} u^{\prime}(\mu+\sigma x) d F} \\
& \geq(>) 0 \quad \text { as } u^{\prime}>0, u^{\prime \prime} \leq(<) 0 \\
& \leq(<) 0 \quad \text { as } u^{\prime}>0, u^{\prime \prime} \geq(>) 0 .
\end{aligned}
$$

The above proposition can be easily extended to include the situation in which $u^{\prime} \geq 0$ and $u^{\prime \prime} \leq 0$ and the situation in which $u^{\prime} \geq 0$ and $u^{\prime \prime} \geq 0$ with the condition $\operatorname{Prob}\left(u^{\prime}>\right.$ $0)>0$. It may be rewritten as the indifference curve $C_{\alpha}$ is convex upwards for risk averters, concave downwards for risk lovers, and horizontal for risk neutral investors.

In addition, we note that Tobin [37] had proven the above proposition on the quadratic utility functions with the normality assumption for the distributions of the return. Our proposition is then an extension of Tobin [37] results to include the general utility functions, as well as the distributions in the location and scale family as in Meyer's paper. Furthermore, since Sinn [34] also obtained similar results for risk averters, our proof is an alternative to the results reported by Tobin and Sinn.

Levy [16] stated the first-degree stochastic dominance (FSD), the second-degree stochastic dominance (SSD), and the mean-variance (MV) rules (Levy called it meanstandard deviation rule); and defined the FSD, SSD, and MV efficient sets (see Levy for the detailed definitions). He also extended Meyer's results to prove that the first- and second-degree stochastic dominance efficient sets are equal to mean-variance efficient set under certain conditions and showed the relationships between the support of the seed random variable $X$ and the parameters in the two linear functions $Y_{i}$ and $Y_{j}$ of $X$ in the following property (Levy termed it as "proposition" in his paper).

Property 2.4. Let $X$ be a random variable with a finite mean and variance, but with no further restriction on its distribution, and let $Y_{i}$ and $Y_{j}$ differ from $X$ by location and scale parameters, such that $Y_{i}=\alpha_{i}+\beta_{i} X, Y_{j}=\alpha_{j}+\beta_{j} X$. The support of $X$ is $[a, b]$. Then

(1) $Y_{i}$ and $Y_{j}$ are in the MV-efficient set for all nondecreasing preferences if and only if

$$
a<\frac{\alpha_{j}-\alpha_{i}}{\beta_{i}-\beta_{j}} .
$$


6 Stochastic dominance theory for location-scale family

(2) (a) If $Y_{i}$ dominates $Y_{j}$ in $\mathrm{MV}$, then such dominance exists in expected utility (EU) for all risk-averse investors with no additional restriction on $F(X)$.

(b) However, a dominance in EU for all nondecreasing $U$ exists, if and only if

$$
b \leq \frac{\alpha_{i}-\alpha_{j}}{\beta_{j}-\beta_{i}}
$$

If (2.12) holds, no dominance by MV implies no dominance for all nondecreasing $U$ and also no dominance for all nondecreasing concave $U$. If (2.12) holds and (2.13) does not hold, the MV- and EU-efficient sets are identical when risk aversion is assumed. If both (2.12) and (2.13) hold, the MV- and EU-efficient sets are identical for all nondecreasing preference $U$.

Next, we study the relationships among the efficient sets for the FSD, SSD, and MV rules for the location-scale family, and the validity of the above property in Levy. Letting $\mathscr{D}_{\mathrm{FSD}}$, $\mathscr{D}_{\mathrm{SSD}}$, and $\mathscr{D}_{\mathrm{MV}}$ be the FSD efficient set, the SSD efficient set, and the MV efficient set, respectively, we obtain the following proposition.

Proposition 2.5. For any location-scale family,

(1) $\mathscr{D}_{\mathrm{SSD}} \subset \mathscr{D}_{\mathrm{FSD}}$;

(2) $\mathscr{D}_{\mathrm{SSD}} \subset \mathscr{D}_{\mathrm{MV}}$; and

(3) (a) $\mathscr{D}_{\mathrm{MV}}-\mathscr{D}_{\mathrm{FSD}} \neq \varnothing$, and

(b) $\mathscr{D}_{\mathrm{FSD}}-\mathscr{D}_{\mathrm{MV}} \neq \varnothing$.

Proof. Since $X \succ_{1} Y \Rightarrow X \succ_{2} Y$, we obtain part (1) of Proposition 2.5. The following is a simple example to show that $\mathscr{D}_{\mathrm{SSD}} \neq \mathscr{D}_{\mathrm{FSD}}$.

Example 2.6. $Y=\beta X$, where $0<\beta<1$ and $E(X)=0$.

In this example, $Y \succ_{2} X$ but $X$ and $Y$ do not dominate each other in the sense of FSD. Hence, $(X, Y) \in \mathscr{D}_{\mathrm{FSD}}$ but $(X, Y) \notin \mathscr{D}_{\mathrm{SSD}}$. Thus, part (1) of the proposition holds.

Applying Hadar and Russell [10, Theorem 4], Tesfatsion [36, Theorem 1'], or Li and Wong [20, Theorem 8b], we find that $\mathscr{D}_{\text {SSD }}$ is a subset of $\mathscr{D}_{M V}$. To show that $\mathscr{D}_{S S D}$ is a proper subset of $\mathscr{D}_{\mathrm{MV}}$, we use the following example.

Example 2.7. Let $X$ be the seed random variable with support $[a, b]=[0,1]$, let $Y_{i}=$ $\alpha_{i}+\beta_{i} X$, and let $Y_{j}=\alpha_{j}+\beta_{j} X$, and set $\beta_{i}>\beta_{j}>0$ and $\alpha_{i}=\alpha_{j}+\beta_{i}-\beta_{j}$.

In this example, $\left(Y_{i}, Y_{j}\right) \in \mathscr{D}_{\mathrm{MV}}$ but $\left(Y_{i}, Y_{j}\right) \notin \mathscr{D}_{\mathrm{SSD}}$. Hence, $\mathscr{D}_{\mathrm{SSD}}$ is a proper subset of $\mathscr{D}_{\text {MV }}$ and thus part (2) of the proposition holds.

Example 2.7 can also be used to prove (3a). In this example, $\left(Y_{i}, Y_{j}\right) \in \mathscr{D}_{\mathrm{MV}}$ but $\left(Y_{i}, Y_{j}\right) \notin \mathscr{D}_{\mathrm{FSD}}$. Hence, (3a) holds.

One can also easily postulate that Example 2.6 can be used to show (3b) as $(X, Y) \in$ $\mathscr{D}_{\mathrm{FSD}}$ but $(X, Y) \notin \mathscr{D}_{\mathrm{MV}}$.

It is well established that the FSD efficient set is equivalent to the EU efficient set for all nondecreasing preference structures $U$, the SSD efficient set is equivalent to the EU efficient set for all nondecreasing concave $U$; see, for example, Hanoch and Levy [12], Hadar and Russell [10], Meyer [24], and Li and Wong [20]. From part (1) of the above 
proposition, we know that the SSD efficient set is a subset of the FSD efficient set. Hence, we can define a complement of the SSD efficient set within the FSD efficient set, denoted by $\mathscr{D}_{S S D}^{c}$, to be the efficient set for all nondecreasing preference $U$ but not for any nondecreasing concave $U$. We have

$$
\mathscr{D}_{\mathrm{FSD}}=\mathscr{D}_{\mathrm{SSD}} \cup \mathscr{D}_{\mathrm{SSD}}^{c}
$$

and $\mathscr{D}_{S S D}^{c}$ is not an empty set. In the proof of parts (2) and (3) in the above proposition, we simply utilize $\left(Y_{i}, Y_{j}\right) \in \mathscr{D}_{\mathrm{SSD}}^{c}$ such that the results hold.

Lastly, we valuate the validity of Levy's property. It is easy to find that Example 2.7 in the above can be used to show that parts (1) and (2b) in Levy's property may not hold. In this example, we illustrate that $\left(Y_{i}, Y_{j}\right) \in \mathscr{D}_{\text {MV }}$ but (2.12) does not hold as

$$
\frac{\alpha_{j}-\alpha_{i}}{\beta_{i}-\beta_{j}}=\frac{\alpha_{j}-\alpha_{j}-\beta_{i}+\beta_{j}}{\beta_{i}-\beta_{j}}=-1<a .
$$

This shows that part (1) in Levy's property may not hold in $\mathscr{D}_{S S D}^{c}$. Additionally, we find that $Y_{i} \succ_{1} Y_{j}$. Applying Li and Wong [20, Theorem 7], we have $E\left[U\left(Y_{i}\right)\right]>E\left[U\left(Y_{j}\right)\right]$ for any nondecreasing $U$ and thus, there exists a dominance in EU for all nondecreasing $U$. However, as

$$
\frac{\alpha_{i}-\alpha_{j}}{\beta_{j}-\beta_{i}}=-1<b
$$

thus inequality in (2.13) does not hold, implying that part (2b) in Levy's property may not hold.

We now give another example in which (2.12) holds but $\left(Y_{i}, Y_{j}\right) \notin \mathscr{D}_{\mathrm{MV}}$ as shown in the following.

Example 2.8. Let $X$ be the seed random variable with support $[a, b]=[0,1]$, let $Y_{i}=$ $\alpha_{i}+\beta_{i} X$, and let $Y_{j}=\alpha_{j}+\beta_{j} X$, and set $\beta_{i}>\beta_{j}>0$ and $\alpha_{j}=\alpha_{i}+\beta_{i}-\beta_{j}$.

In this example, since $\beta_{i}>\beta_{j}>0$ and $\alpha_{j}>\alpha_{i}$, we have $\left(Y_{i}, Y_{j}\right) \notin \mathscr{D}_{\mathrm{MV}}$. However,

$$
\frac{\alpha_{j}-\alpha_{i}}{\beta_{i}-\beta_{j}}=\frac{\alpha_{i}+\beta_{i}-\beta_{j}-\alpha_{i}}{\beta_{i}-\beta_{j}}=1>a
$$

and hence (2.12) holds. This leads to our conclusion that part (1) of Levy's property does not hold in this example. However, in this example, we find that

$$
\frac{\alpha_{i}-\alpha_{j}}{\beta_{j}-\beta_{i}}=1 \geq b
$$

and hence (2.13) holds and it is easy to show that $Y_{i} \succ_{1} Y_{j}$. In this connection, part (2b) of Levy's property is valid in this example. Another trivial example in which part (2b) does not hold is the following.

Example 2.9. We set $\alpha_{i}>\alpha_{j}$ and $\beta_{i}=\beta_{j}$.

In this example, $Y_{i} \succ_{1} Y_{j}$ and hence there exists a dominance in EU for all nondecreasing $U$ but (2.13) does not hold. 


\section{Concluding remarks}

Meyer [25] contributed to the theory of mean-variance criterion by extending the theory to include the comparison among distributions that differ only by location and scale parameters as well as to include the general utility functions with only convexity or concavity restrictions. Levy [16] extended Meyer's results by introducing some relationships between the stochastic-dominance and the mean-variance efficient sets. However, Meyer [26] commented that Levy's findings is an application of the principle that segments of efficient sets cannot have slopes which are greater (smaller) than the highest (least) sloped indifference curve and commented that those portions of the MV-efficient set which are either too flat or too steeply sloped are not EU efficient.

We first make some comments on Meyer's paper and extend the results from Tobin [37] that the indifference curve is convex upward for risk averters, concave downwards for risk lovers, and horizontal for risk neutral investors to include the general conditions as stated in Meyer [25]. We then comment on Levy's findings and show that the relationships in Levy's property do not hold in certain situations. We further explore the relationships among the first- and second-degree stochastic dominance efficient sets and the mean-variance efficient set to show that they are not equal to one another. We check the literature on the subject and conclude that the results in our paper are still new and we hope that our results would be able to contribute to the existing literature.

Further extensions of the theory developed in this paper, future work could extend our efforts to link stochastic dominance to mean-variance criterion developed by Markowitz [21], Tobin [37], and Sharpe [33] for location-scale family. As the theory developed by Meyer and Levy, and in this paper mainly concerns only risk averters, it would also be worthwhile to extend it to risk lovers (see, e.g., Hammond [11], Meyer [24], Hershey and Schoemaker [13], Stoyan [35], Myagkov and Plott [27], Wong and Li [44], Post [28], Anderson [1], and Post and Levy [30]) and to investors with S-shaped or reverse S-shaped utility functions (see, e.g., Kahneman and Tversky [14], Tversky and Kahneman [38], Levy and Wiener [19], and Levy and Levy [17, 18]). Another area of extension is to extend our theory to a variable of loss (see, e.g., Weeks and Wingler [41], Weeks [40], Post and Diltz [29], and Dillinger et al. [5]). In addition, the theory developed in this paper could be applied to many different areas in business, economics, and finance. For example, one could easily incorporate our approach to explain well-known financial anomalies (see, e.g., McNamara [23], Wong and Bian [42], Post [28], Post and Levy [30], Kuosmanen [15], and Fong et al. [9]) and to model investment risk (see, e.g., Matsumura et al. [22], Doumpos et al. [6], Wong and Chan [43], Fong and Wong [8], and Broll et al. [3]).

\section{Acknowledgments}

The author is grateful to professor Mahyar Amouzegar and anonymous referees for their substantive comments that have significantly improved this manuscript. My deepest thanks are given to Thomas Kwok Keung Au, Bin Cheng, and Song Yan for their helpful assistance and comments. The author would also like to thank Robert B. Miller and Howard E. Thompson for their continuous guidance and encouragement. 


\section{References}

[1] G. Anderson, Toward an empirical analysis of polarization, Journal of Econometrics 122 (2004), no. $1,1-26$.

[2] D. P. Baron, On the utility theoretic foundations of mean-variance analysis, Journal of Finance 32 (1977), no. 5, 1683-1697.

[3] U. Broll, J. E. Wahl, and W.-K. Wong, Elasticity of risk aversion and international trade, Economics Letters 91 (2006), no. 1, 126-130.

[4] K. V. Chow, Marginal conditional stochastic dominance, statistical inference, and measuring portfolio performance, Journal of Financial Research 24 (2001), no. 2, 289-307.

[5] A. M. Dillinger, W. E. Stein, and P. J. Mizzi, Risk averse decisions in business planning, Decision Sciences 23 (1992), no. 4, 1003-1008.

[6] M. Doumpos, S. Zanakis, and C. Zopounidis, Multicriteria preference disaggregation for classifcation problems with an application to global investing risk, Decision Sciences 32 (2001), no. 2, 333-385.

[7] M. S. Feldstein, Mean-variance analysis in the theory of liquidity preference and portfolio selection, Review of Economics Studies 36 (1969), no. 1, 5-12.

[8] W. M. Fong and W.-K. Wong, The modified mixture of distributions model: a revisit, Annals of Finance 2 (2006), no. 2, 167-178.

[9] W. M. Fong, W.-K. Wong, and H. H. Lean, International momentum strategies: a stochastic dominance approach, Journal of Financial Markets 8 (2005), no. 1, 89-109.

[10] J. Hadar and W. R. Russell, Stochastic dominance and diversification, Journal of Economic Theory 3 (1971), no. 3, 288-305.

[11] J. S. Hammond, Simplifying the choice between uncertain prospects where preference is nonlinear, Management Science 20 (1974), no. 7, 1047-1072.

[12] G. Hanoch and H. Levy, Efficiency analysis of choices involving risk, Review of Economic Studies 36 (1969), no. 3, 335-346.

[13] J. C. Hershey and P. J. H. Schoemaker, Risk taking and problem context in the domain of losses: an expected utility analysis, Journal of Risk and Insurance 47 (1980), no. 1, 111-132.

[14] D. Kahneman and A. Tversky, Prospect theory: an analysis of decision under risk, Econometrica 47 (1979), no. 2, 263-291.

[15] T. Kuosmanen, Efficient diversification according to stochastic dominance criteria, Management Science 50 (2004), no. 10, 1390-1406.

[16] H. Levy, Two-moment decision models and expected utility maximization: comment, American Economic Review 79 (1989), no. 3, 597-600.

[17] M. Levy and H. Levy, Prospect theory: much ado about nothing?, Management Science 48 (2002), no. $10,1334-1349$.

[18] H. Levy and M. Levy, Prospect theory and mean-variance analysis, Review of Financial Studies 17 (2004), no. 4, 1015-1041.

[19] H. Levy and Z. Wiener, Stochastic dominance and prospect dominance with subjective weighting functions, Journal of Risk and Uncertainty 16 (1998), no. 2, 147-163.

[20] C.-K. Li and W.-K. Wong, Extension of stochastic dominance theory to random variables, RO Recherche Opérationnelle 33 (1999), no. 4, 509-524.

[21] H. M. Markowitz, Portfolio selection, Journal of Finance 7 (1952), no. 1, 77-91.

[22] E. M. Matsumura, K. W. Tsui, and W.-K. Wong, An extended multinomial-Dirichlet model for error bounds for dollar-unit sampling, Contemporary Accounting Research 6 (1990), no. 2, 485500.

[23] J. R. McNamara, Portfolio selection using stochastic dominance criteria, Decision Sciences 29 (1998), no. 4, 785-801.

[24] J. Meyer, Second degree stochastic dominance with respect to a function, International Economic Review 18 (1977), no. 2, 477-487. 
10 Stochastic dominance theory for location-scale family

[25] _ Two-moment decision models and expected utility maximization, American Economic Review 77 (1987), no. 3, 421-430.

[26] _ Two-moment decision models and expected utility maximization: reply, American Economic Review 79 (1989), no. 3, 603.

[27] M. Myagkov and C. R. Plott, Exchange economies and loss exposure: experiments exploring prospect theory and competitive equilibria in market environments, American Economic Review 87 (1997), no. 5, 801-828.

[28] T. Post, Empirical tests for stochastic dominance efficiency, The Journal of Finance 58 (2003), no. 5, 1905-1931.

[29] G. V. Post and J. D. A. Diltz, A stochastic dominance approach to risk analysis of computer systems, MIS Quarterly 10 (1986), no. 4, 362-375.

[30] T. Post and H. Levy, Does risk loving drive asset prices? a stochastic dominance analysis of aggregate investor preferences and beliefs, Review of Financial Studies 18 (2005), no. 3, 925-953.

[31] M. Rothschild and J. E. Stiglitz, Increasing risk. I. A definition, Journal of Economic Theory 2 (1970), no. 3, 225-243.

[32] _ Increasing risk. II. Its economic consequences, Journal of Economic Theory 3 (1971), no. $1,66-84$.

[33] W. F. Sharpe, A simplified model for portfolio analysis, Management Science 9 (1963), no. 2, 277293.

[34] H.-W. Sinn, Economic Decisions under Uncertainty, Studies in Mathematical and Managerial Economics, vol. 32, North-Holland, Amsterdam, 1983.

[35] D. Stoyan, Comparison Methods for Queues and Other Stochastic Models, Wiley Series in Probability and Mathematical Statistics: Applied Probability and Statistics, John Wiley \& Sons, Chichester, 1983.

[36] L. Tesfatsion, Stochastic dominance and the maximization of expected utility, Review of Economic Studies 43 (1976), no. 2, 301-315.

[37] J. Tobin, Liquidity preference as behavior towards risk, Review of Economics Studies 25 (1958), no. $2,65-86$.

[38] A. Tversky and D. Kahneman, Advances in prospect theory: cumulative representation of uncertainty, Journal of Risk and Uncertainty 5 (1992), no. 4, 297-323.

[39] J. von Neumann and O. Morgenstern, Theory of Games and Economic Behavior, Princeton University Press, New Jersey, 1944.

[40] J. K. Weeks, Stochastic dominance: a methodological approach to enhancing the conceptual foundations of operations management theory, Academy of Management Review 10 (1985), no. 1, $31-38$

[41] J. K. Weeks and T. R. Wingler, A stochastic dominance ordering of scheduling rules, Decision Sciences 10 (1979), no. 2, 245-257.

[42] W.-K. Wong and G. Bian, Robust estimation in capital asset pricing model, Journal of Applied Mathematics and Decision Sciences 4 (2000), no. 1, 65-82.

[43] W.-K. Wong and R. H. Chan, On the estimation of cost of capital and its reliability, Quantitative Finance 4 (2004), no. 3, 365-372.

[44] W.-K. Wong and C.-K. Li, A note on convex stochastic dominance, Economics Letters 62 (1999), no. 3, 293-300.

[45] Y. Zhao and W. Ziemba, A dynamic asset allocation model with downside risk control, Journal of Risk 3 (2000), no. 1, 91-113.

Wing-Keung Wong: Risk Management Institute and Department of Economics, National University of Singapore, 1 Arts Link, Singapore 117570

E-mail address: ecswwk@nus.edu.sg 


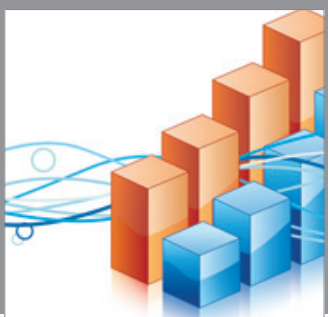

Advances in

Operations Research

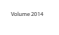

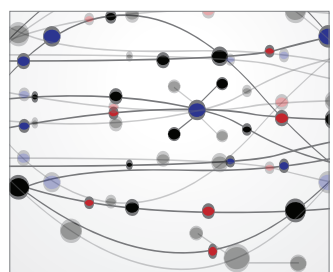

\section{The Scientific} World Journal
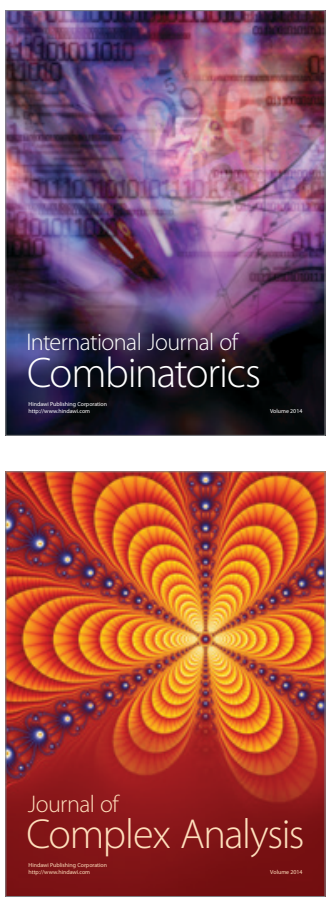

International Journal of

Mathematics and

Mathematical

Sciences
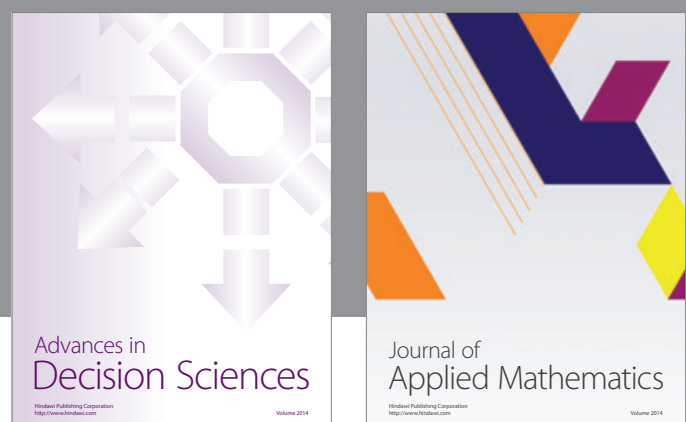

Journal of

Applied Mathematics
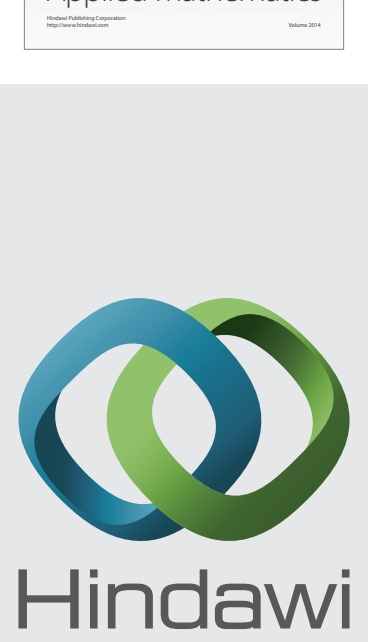

Submit your manuscripts at http://www.hindawi.com
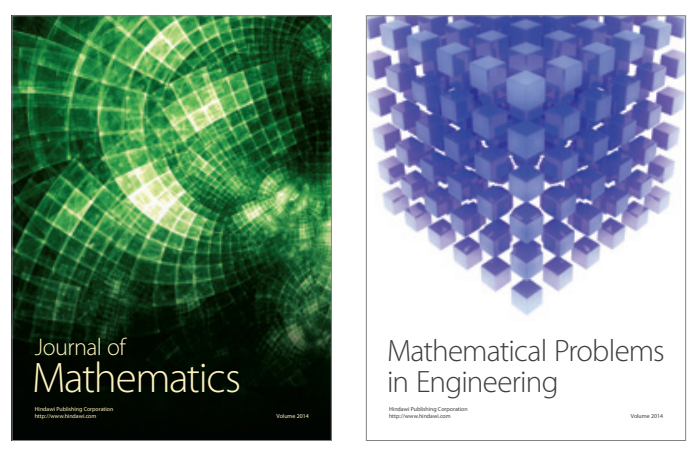

Mathematical Problems in Engineering
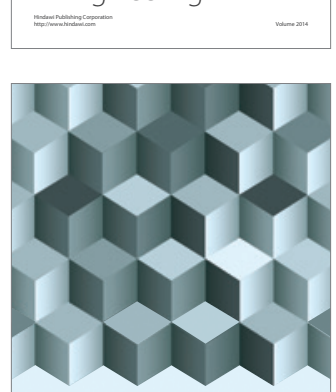

Journal of

Function Spaces
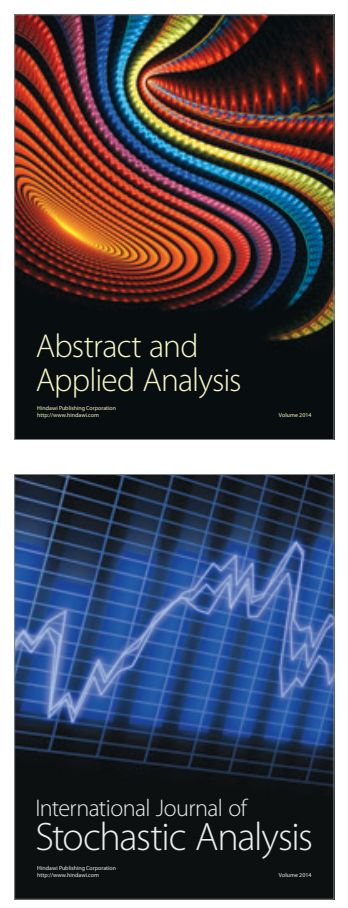

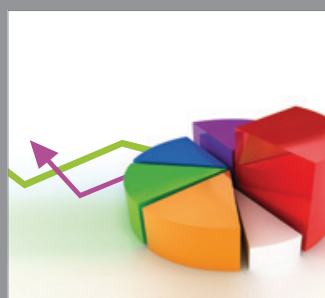

ournal of

Probability and Statistics

Promensencen
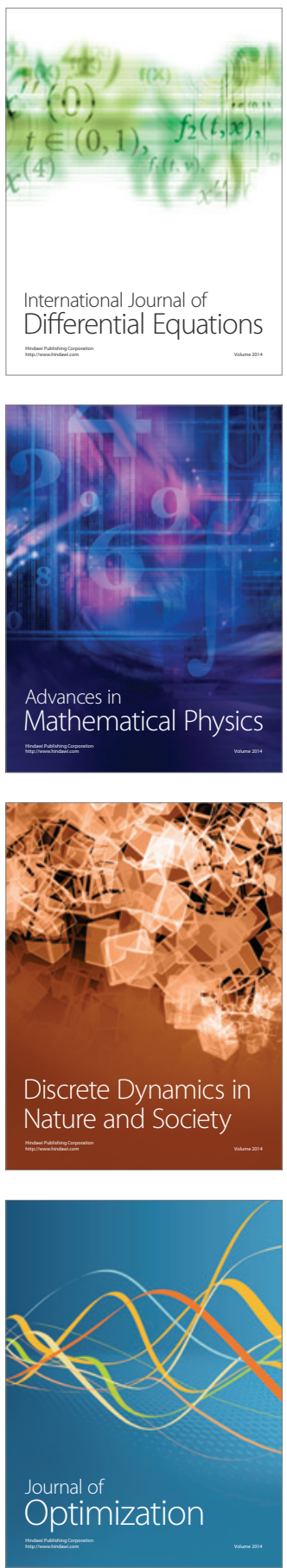